\title{
STATISTICAL MECHANICS MODEL FOR ORIENTATIONAL MOTION OF TWO-DIMENSIONAL RIGID ROTATOR
}

Malo, J.O.

\author{
Department of Physics, University of Nairobi, P.O. Box 30197 NAIROBI - Kenya
}

\begin{abstract}
A comparison of thermodynamics properties of two-dimensional linear rigid rotator is presented in the absence, and presence of an impressed weak electric field at low and high temperatures. It is shown that specific heat and entropy fall off rapidly at low temperatures. At ultra low temperatures, the entire normal entropy is lost and we have complete ordering by the impressed field. In the classical limit it is shown that there is a temperature dependent polarizability $\frac{1}{2} \mu^{2} / K T$.
\end{abstract}

\section{INTRODUCTION}

We first present some formalism of statistical mechanics and thermodynamics ${ }^{[1,2]}$. Let us imagine for a moment that our two-dimensional dipoles are tacked down such that there is no translational motion and that they are well separated so that they do not exert direct forces on each other. Imagine further that there is a buffer gas of neutral molecules that constitutes a reservoir at temperature $T$. Then the probability that a dipole will be found in the $j^{\text {th }}$ energy state $\left(E_{j}\right)$ is

$$
P_{j}=e^{-\beta E_{j}} / \sum_{j} e_{j}^{-\beta E}
$$

This is a canonical distribution where $\beta=1 / K T$ and $K$ is the Boltzmann constant

It is important to note that the assumption here is that each dipole is in a weak contact with the reservoir. For example, a dipole might be hit impulsively by the molecules of the reservoir ${ }^{[3]}$. These collisions serve to send the dipole into one or another of its energy states. But most of the time the dipole is alone in the external field ${ }^{[4]}$. The average energy of a buffer molecule in two-dimension is

$2 x\left(\frac{K T}{2}\right)$. However some have more and some have less energy which gives rise to the energy distribution of the dipole molecules ${ }^{[5]}$. The average energy $\bar{E}(\beta, F)$ of a dipole is

$\bar{E}(\beta, F)=\sum_{j} E_{j}(F) e^{-\beta E_{j}^{(F)}} / \sum_{j} e^{-\beta E_{j}(F)}$

where $F$ is the impressed field.

The specific heat per dipole at constant external field is

$C_{F}=\left(\frac{\partial \bar{E}}{\partial T}\right)_{F}$

We note here that if we have $\rho$ as the dipole per unit volume, we first multiply extensive quantities by $\rho$ to get, for example, specific heat per unit volume $C_{V}$.

Now the $\bar{E}(\beta, F)$ quantity is to be identified with internal energy. Thus entropy $S$ per dipole for the canonical distribution is defined as

$S=-K \Sigma P_{j} \ln P_{j}=S(\beta, F)$

Finally the free energy $A(\beta, F)$ is defined as

$$
A(\beta, F)=-\frac{1}{\beta} \ln Z
$$




$$
Z(\beta, F)=e^{-\beta H}=\sum_{j} e^{-E_{j}(\beta, F)}
$$

and $Z(\beta, F)$ is the partition function of the system in the presence of an impressed electric field.

We can quickly check that the above identifications agree with the well known thermodynamic relations. For example the Gibbs-Helmholtz equation is

$$
\bar{E}=-T^{2}\left(\frac{\partial A}{\partial T}\right)_{F}
$$

and we have the usual relation

$$
A=\bar{E}-T S
$$

To check this we note that in equilibrium

$$
\begin{aligned}
S & =-K \sum_{j} P_{j} \ln P_{j}=-K \sum_{j} \frac{e^{-\beta E_{j}}}{Z}\left\{-\beta E_{j}-\ln Z\right\} \\
S & =-K \sum_{j} P_{j} \ln P_{j}=-K \sum_{j} \frac{e^{-\beta E_{j}}}{Z}\left\{-\beta E_{j}-\ln Z\right\} \\
S & =K \beta \bar{E}+K \ln Z \\
& =K \beta \bar{E}+K(-\beta A)
\end{aligned}
$$

This paper is organized as follows. Section 2 is devoted to the treatment of the two-dimensional rotator in the absence and presence of the impressed fields at low and high temperature limits. The classical limit of statistics is discussed in section 3 and it is shown that there is a temperature dependant polarizability. In section 4 a study of the way that the classical limit emerges from the formulae of quantum statistics is presented.

\section{TWO DIMENSIONAL RIGID ROTATOR}

Our main objective here is to compute certain thermodynamic quantities for the two-dimensional dipole [6]. We shall therefore focus attention on the partition function for a given dipole

$$
Z=\sum_{j} e^{-\beta E_{j}}
$$

\section{Absence of External Field}

In the absence of the field, we have immediately

$Z^{o}=\sum_{m=-\infty}^{-\infty} e^{-\beta} \frac{h^{2}}{2 I} m^{2}$

This sum can be done in the classical limit where $\frac{\beta h^{2}}{2 I}\langle\langle 1$, that is, $K T\rangle\rangle h^{2} / 2 I$. Here $h$ is Planck's constant and $I$ is the moment of inertia of a linear rotator. Thus, the successive terms in the sum differ only slightly from each other except for high values of $m$, where the contributions rapidly become small.

Denoting $\alpha \equiv \beta \frac{\hbar^{2}}{2 I}$, where $\sqrt{\alpha}$ is the thermal de Broglie angle, we have

$$
Z^{o}=\sum_{m=-\infty}^{+\infty} e^{-\alpha m^{2}}
$$

For the classical limit, we treat $m$ as continuous then

$Z^{o} \Rightarrow \int_{-\infty}^{+\infty} e^{-\alpha m^{2}} d m=\sqrt{\frac{\pi}{\alpha}}$

Thus the average internal energy of a dipole

$$
\begin{aligned}
& \bar{E}=\frac{1}{Z^{o}} \sum_{m=-\infty}^{+\infty} \frac{h^{2} m^{2}}{2 I} e^{-\alpha m^{2}} \\
& \Rightarrow \frac{h^{2}}{2 I} \frac{1}{\alpha^{3 / 2}} \frac{\int_{-\infty}^{+\infty} x^{2} e^{-x^{2}} d x}{\sqrt{\frac{\pi}{\alpha}}} \Rightarrow \frac{K T}{2}
\end{aligned}
$$

and the specific heat is $K / 2$

The free energy is given by

$A^{0}=-K T \ln Z^{0}$ 
and thus

$$
A_{\text {classical }}^{0} \Rightarrow-\frac{K T}{2}\{\ln \pi-\ln \alpha\}
$$

The entropy $S^{0}$ is given by

$$
\begin{aligned}
& S_{\text {classical }}^{0}=-K \beta^{2} \frac{\partial A^{0}}{\partial \beta} \Rightarrow \\
& -\frac{K \beta^{2}}{2} \frac{\partial}{\partial \beta}\left\{\frac{\ln \pi}{\beta}-\ln \beta+\frac{\ln \left(\frac{2 I}{\hbar^{2}}\right)}{\beta}\right\} \\
& =\frac{K}{2} \ln \left(\pi \frac{2 I K T}{\hbar^{2}}\right)
\end{aligned}
$$

Thus even though this is classical, it contains $\hbar^{2}$, which enters as an additive constant fixed by quantum theory, and it is clear from (17) that entropy increases with temperature.

At low temperatures $\alpha\rangle\rangle 1$, and we must therefore use the quantum expression where only the lowest and first energy levels are populated, thus

$Z^{o} \Rightarrow 1+2 e^{-\alpha}+\ldots \ldots \ldots \ldots \ldots . . .=1+2 e^{-\frac{\beta \hbar^{2}}{2 \mathrm{I}}}$

$\ln Z^{0} \Rightarrow 2 e^{-\alpha}$

simply because $\ln (1+x) \simeq x-\frac{x^{2}}{2}+\frac{1}{3} x^{3}+\ldots \ldots$ for $-1 \leq x \leq 1$, and taking only the first term in the series expansion. Thus the free energy is given by

$A^{0}=-\frac{1}{\beta} 2 e^{-\beta \hbar^{2} / 2 I}$

The average internal energy $\bar{E}$

$$
\begin{aligned}
& \bar{E} \Rightarrow \frac{\frac{\hbar^{2}}{2 I} 2 e^{-\beta \hbar^{2} / 2 I}}{1+2 e^{-\beta^{2} / 2 I}} \\
& \simeq \frac{\hbar^{2}}{\mathrm{I}} e^{-\beta \hbar^{2} / 2 I}
\end{aligned}
$$

The specific heat

$$
C \Rightarrow K \beta^{2} \frac{\hbar^{2}}{I} \cdot \frac{\hbar^{2}}{2 I} e^{-\beta \frac{\hbar^{2}}{2 I}}=2 K\left(\frac{\hbar^{2}}{2 \mathrm{I} K T}\right)^{2} e^{-\beta^{\frac{\hbar^{2}}{2 \mathrm{I}}}}
$$

It is trivial to show that (21) vanishes rapidly as $T \rightarrow 0$. This is attributable to the scarcity of available levels due to quantization. The rapid fall of the specific heat at low temperatures is always attributable to the scarcity of the low-lying states.

\section{Dipole in an External Field}

Turning to the behaviour in an external field $F(t)$, we note that it is not as easy as one might first think. First of all it is necessary to know the energy levels in the presence of the field before we can evaluate

$$
Z=\sum_{m=-\infty}^{+\infty} e^{-\beta E_{m}(F)}
$$

The strength of the interaction is measured by the dimensionless ratio $\mu F /\left(\hbar^{2} / 2 I\right)$, where $\mu$ is the dipole moment operator. Simple perturbation theory holds for $\mu F\left\langle\left\langle\hbar^{2} / 2 I\right.\right.$. This depends crucially on Planck's constant being nonzero. In statistical mechanics, there is the second dimensionless parameter $\alpha=\frac{\hbar^{2}}{2 I} \beta=\frac{\hbar^{2}}{2 I K T}$. We will hence limit our consideration to the weak external field $\left(\mu F \ll \hbar^{2} / 2 I\right)$, for which we apply quantum mechanical perturbation theory in the limit of low and high temperature regions.

Let us introduce the following parameter $\lambda=\mu F \cdot \frac{2 I}{h^{2}}$.

When $\lambda \ll<1$, in lowest non-vanishing order, the energy levels are

$$
E_{m}(F)=\frac{\hbar^{2} m^{2}}{2 I}+\frac{\hbar^{2}}{2 I} \cdot \frac{\lambda^{2}}{2} \cdot \frac{1}{4 m^{2}-1}+\ldots \ldots \ldots
$$

If the perturbation theory is valid for the lowest $(m=0)$ level, it is even better for the excited states. 
Let us now make some nontrivial estimates. For a small molecule, the dipole moment is of the order of the electronic charge $e=4.8 \times 10^{-10}$ e.s.u multiplied by a typical atomic separation of $1 \AA$ in a molecule. For $\mathrm{HCl}, \mu=1.03 \times 10^{-18}$ in e.s.u. Since 1 e.s.u $=300$ volts, a field of 300 volts $/ \mathrm{cm}$ has $\mu F \simeq 10^{-18}$ ergs and in general $\mu F=10^{-18}\left(\frac{F_{v}}{300}\right)$ ergs, where $F_{v}$ is the field in volts per centimeter. $\mathrm{In} \mathrm{HCl}$ $h^{2} / 2 I \simeq 2 \times 10^{-15}$ ergs, hence $\lambda=\mu F \frac{2 I}{\hbar^{2}}=\frac{10^{-3}}{2}\left(\frac{F_{v}}{300}\right)$. This value of $\lambda$ is much less than unity for reasonable fields. We therefore have for the weak field case

$$
Z \simeq \sum_{m=-\infty}^{+\infty} e^{-\frac{\beta h^{2}}{2 \mathrm{I}}}\left(m^{2}+\frac{\lambda^{2}}{2} \frac{1}{4 m^{2}-1}\right)
$$

The above partition function serves two distinct cases, i.e. the low and the high temperature regions.

\section{(i) Low temperatures}

Here

$\left.\left.\alpha=\frac{h^{2}}{2 I K T}=\frac{h^{2}}{2 I} \beta\right\rangle\right\rangle 1$

For HCl molecule, $\frac{h^{2}}{2 I} \simeq 2 \times 10^{-15}$ ergs so that $\alpha=\frac{15.2}{T}$ where $T$ is in degrees Kelvin. Thus for $T<5^{\circ} K$ our condition is well satisfied and only the lowest $(m=0)$ and the first set of the energy levels are populated. Consequently, the partition function takes the form

$$
z \simeq e^{\alpha^{\frac{\lambda^{2}}{2}}}\left\{1+2 e^{-\alpha} e-\frac{\alpha \lambda^{2}}{4}+\ldots \ldots\right\}
$$

The free energy is given by

$$
A \simeq-\frac{\hbar^{2}}{16 I} \lambda^{2}-\frac{2}{\beta} e^{-\alpha} e^{\frac{\alpha \lambda^{2}}{4}}
$$

and the entropy of the system is

$$
S \simeq 2 K e^{-\alpha} e^{-\frac{\alpha \lambda^{2}}{4}}+K \alpha\left(1+\frac{\lambda^{2}}{4}\right) e^{-\alpha} e^{-\alpha^{\frac{\lambda^{2}}{4}}}
$$

We note here that when $\lambda \simeq 0$ (very weak field) entropy is given by

$$
S=K(2+\alpha) e^{-\alpha}
$$

and for a finite $\lambda$, the change in entropy $\Delta S$ is given by

$$
\begin{aligned}
\Delta S= & 2 K e^{-\alpha}\left(e^{-\frac{\alpha \lambda^{2}}{4}}-1\right)+ \\
& k \alpha e^{-\alpha}\left(e^{-\frac{a \lambda^{2}}{4}}-1\right)+K^{\alpha-\frac{\lambda^{2}}{4}} e^{-\alpha} e^{\frac{\alpha \lambda^{2}}{4}}
\end{aligned}
$$

There are now two subcases: The first one is when $\frac{\alpha \lambda^{2}}{4}<<1$, then

$\frac{1}{4} \frac{\hbar^{2}}{2 I K T}(\mu F)^{2}\left(\frac{2 I}{\hbar^{2}}\right)^{2}=\frac{1}{4} \frac{\mu F}{K T}\left(\mu F \frac{2 I}{\hbar^{2}}\right) \ll<1$

or

$$
\frac{1}{4} \frac{\mu F}{K T} \lambda \ll<1
$$

For HCl, $\frac{\mu F}{K T}=\frac{10^{-4}}{1.4 T}\left(\frac{F v}{300}\right)$ so that $\frac{\alpha \lambda^{2}}{4}\langle\langle 1$ is the usual case unless the temperature is well below $1^{\circ} \mathrm{K}$. In that case then we expand the exponential to get

$$
\Delta S=K e^{-\beta \frac{\hbar^{2}}{2 \mathrm{I}}}\left\{-\frac{\alpha \lambda^{2}}{4}-\frac{\alpha^{2} \lambda^{2}}{2}+\ldots \ldots\right\}
$$

The above expression (31) indicates entropy decreases on ordering by the field. The dominant term is $-\frac{\alpha^{2} \lambda^{2} K}{2} e^{-\alpha}$ and is very small.

The second subcase is at very low temperatures where $\alpha\rangle) 1$, hence for $\mathrm{HCl} \alpha=\frac{15.2}{T}$, we note here that since $\lambda$ is independent of temperature, we can reach this case at ultra low temperatures. Then $S \rightarrow 0$ i.e., the entire normal entropy is lost and we have complete ordering by the impressed field. 


\section{(ii) High temperatures}

Here $\alpha=\beta \frac{h^{2}}{2 I}=\frac{h^{2}}{2 I K T} \ll<1$

We still have the weak field case $\lambda=\mu F \cdot \frac{2 I}{h^{2}}\langle<1$ so the quantum perturbation theory is valid. But now many energy levels are populated and we must sum over all levels in the partition function.

$Z=\sum_{m=-\infty}^{+\infty} e^{-\beta \frac{h^{2}}{2 I}}\left(m^{2}+\frac{\lambda^{2}}{2} \frac{1}{4 m^{2}-1}\right)$

We will however, split off the term $m=0$ in any case since we know that the perturbation shift is anomalous. Thus

$$
Z=e^{+\alpha} \frac{\lambda^{2}}{2}+\sum^{1} e^{-\alpha}\left(m^{2}+\frac{\lambda^{2}}{2} \frac{1}{4 m^{2}-1}\right)
$$

Now that $\alpha$ as well as $\lambda$ are $\langle<1$, we can replace the sum by an integral

$Z \simeq e^{\alpha} \frac{\lambda^{2}}{2}+2 \int_{1}^{\infty} d m e^{-\alpha\left(m^{2}+\frac{\lambda^{2}}{2} \frac{1}{4 m^{2}-1}\right)}$

It is possible to analyse the above integral more carefully, however under our conditions it is more legitimate to expand.

$\left.Z=e^{\alpha} \frac{\lambda^{2}}{2}+2 \int_{1}^{\infty} d m e^{-m^{2}\left(1-\frac{\alpha \lambda^{2} / 2}{4 m^{2}-1}+\ldots \ldots\right.}\right)$

Taking $X=\sqrt{\alpha m}$ we come to

$Z=e^{\alpha \frac{\lambda^{2}}{2}}+\frac{2}{\sqrt{\alpha}} \int_{\sqrt{\alpha}}^{\infty} e^{-x^{2}} d x\left(1-\frac{\alpha^{2} \lambda^{2} / 2}{4 x^{2}-\alpha}\right)$

For a fixed $\alpha$, the change in the partition function due to the applied field is

$\Delta Z=\left(e^{\alpha^{\frac{\lambda^{2}}{2}}}-1\right)-F_{1}(\alpha) \lambda^{2}$ where $F_{1} \alpha$ is a positive function of $\alpha$ expressed as

$$
F_{1}(\alpha)=\frac{2}{\sqrt{\alpha}} \int_{\sqrt{\alpha}}^{\infty} \frac{\alpha^{\frac{1}{2}}}{4 x^{2}-\alpha} e^{-x^{2}} d x
$$

Thus $A \simeq-K T\left\{\ln \left(Z^{0}+\Delta Z\right)\right\}=-K T \ln \frac{\Delta Z}{Z^{0}}$ is of the order $\lambda^{2}$ as is the entropy change. $Z^{0}$ is the partition function in the absence of the external field ( $\$ 2.1)$.

\section{THE CLASSICAL LIMIT OF STATISTICS}

Let us consider macromolecules for which the moment of inertia is hundreds of times that of $\mathrm{HCl}$, so that $\lambda=\mu F \frac{2 I}{\hbar^{2}}\left\langle\left\langle 1\right.\right.$ and $\alpha=\frac{\hbar^{2}}{2 I K T}\langle\langle 1$ at moderate temperatures. We now briefly recall the classical theory and then examine the relationship between classical and quantum statistics in more detail.

The classical analogue of the partition function is

$Z=\frac{1}{2} \int_{o}^{2 \pi} \int_{-\alpha}^{\infty} e^{-\beta} d P_{\theta} d \theta$

Here we use the Hamiltonian description

$H\left(P_{\theta}, \theta\right)=\frac{P_{\theta}^{2}}{2 I}-\mu F \cos \theta$

where $P_{\theta}$ and $\theta$ are the independent variables i.e., canonical momentum and coordinate. The above multiple integral goes over the phase space and it is important to note particularly that the range of integration of $P_{\theta}$ is $-\infty$ to $+\infty$ i.e., the angular momentum can be as large as one likes, and the constant $\hbar$ is introduced for dimensional reasons.

In the present case, it is easy to evaluate the partition function

$$
\begin{aligned}
Z & =\frac{1}{\hbar} \int_{-\infty}^{\infty} e^{-\frac{\beta P_{\theta}^{2}}{2 I}} d P_{\theta} \cdot \int_{o}^{2 \pi} e^{-\beta \mu F \cos \theta} d \theta \\
& =\frac{1}{\hbar} \sqrt{\frac{\pi 2 I}{\beta}} \int_{o}^{2 \pi} e^{-\beta \mu F \cos \theta} d \theta
\end{aligned}
$$


The remaining integral is a Bessel function ${ }^{[7,8]}$

$\int_{0}^{2 \pi} e^{-y \cos \theta} d \theta=2 \pi I_{o}(y)$

where $y=\mu F / K T$

Thus

$I_{o}(y) \Rightarrow \frac{e^{-y}}{\sqrt{2 \pi y}}\left\{1+\frac{1}{8 y}+\frac{9}{2(8 y)^{2}}+\ldots \ldots .\right.$.

for $y\rangle>1$

and

$I_{o}(y) \Rightarrow 1-\frac{1}{4} y^{2}+\ldots$

for $y \ll<1$

We can now go through our previous calculation and first in importance is polarization average per dipole

$$
\begin{aligned}
& P=\frac{\int \mu \cos \theta e^{-\beta H} d P_{\theta} d \theta}{\int e^{-\beta H} d P_{\theta} d \theta} \Rightarrow \\
& \Rightarrow \frac{\int_{0}^{2 \pi} \mu \cos \theta e^{-\beta} \mu F \cos ^{\theta} d P_{\theta} d_{\theta}}{\int_{0}^{2 \pi} e^{-} \beta \mu F \cos \theta d P_{\theta} d_{\theta}} \\
& =-\frac{1}{\beta} \frac{\partial}{\partial F} \ln \left\{\int e^{-\beta \mu F \cos \theta} d \theta\right\} \\
& =-\frac{1}{\beta} \frac{\partial}{\partial F}(\ln Z)
\end{aligned}
$$

The kinetic energy factor in the partition function $Z$ drops out in the differentiation and we obtain

$$
P=-\frac{1}{\beta} \frac{\partial}{\partial F}\left[\ln \mathrm{I}_{o}(y)\right]
$$

For small $y$

$$
P \Rightarrow+\frac{1}{\beta} \frac{\partial}{\partial F}\left(\frac{1}{4} \frac{\mu^{2} F^{2}}{(K T)^{2}}\right)=+\frac{1}{2}\left(\frac{\mu^{2}}{K T}\right) F
$$

so that there is a temperature dependant polarizability

$$
\frac{1}{2} \frac{\mu^{2}}{K T}
$$

$$
\begin{aligned}
& P=-\frac{1}{\beta} \frac{\partial}{\partial F} \ln \left(\frac{e^{y}}{\sqrt{2 \pi y}}\right) \\
& \Rightarrow+\frac{1}{\beta} \frac{\partial}{\partial F} \cdot y \Rightarrow \mu
\end{aligned}
$$

This is the case where thermal motion is unimportant. Thus the free energy is given by

$$
\begin{aligned}
A & =K T \ln Z \\
& =K T \ln \frac{1}{h} \sqrt{\frac{\pi}{\beta} 2 I}-K T \ln \left[2 \pi I_{o}(y)\right]
\end{aligned}
$$

and for weak fields

$$
\begin{aligned}
A \Rightarrow & -\frac{K T}{2} \ln \left(\frac{\pi}{h^{2}} K T-2 I\right) \\
& -K T\left\{\ln (2 \pi)-\frac{1}{4} y^{2}+\ldots \ldots \ldots . . .\right\}
\end{aligned}
$$

Consequently the field dependent part has the following variation,

$$
\Delta A=\frac{1}{4} \frac{(\mu F)^{2}}{K T}+\ldots \ldots \ldots
$$

The internal energy can be computed directly as

$\bar{E}=\frac{\int H e^{-\beta H} d P_{\theta} d \theta}{\int e^{-\beta H} d P_{\theta} d \theta}$

$\Rightarrow \bar{E}=-T^{2}\left(\frac{\partial}{\partial T}\left(\frac{A}{T}\right)\right)_{F}$

In the weak field case, the internal energy change is given by

$$
\begin{aligned}
\Delta \bar{E} & =-T^{2} \frac{(\mu F)^{2}}{4 K}\left(-\frac{2}{T^{3}}\right) \\
& =\frac{1}{2}\left(\frac{\mu F}{K T}\right) \cdot \mu F
\end{aligned}
$$


The entropy, variation is thus given by

$$
\begin{aligned}
\Delta S & =\frac{1}{T}\left\{\frac{1}{2} \frac{\mu F}{K T} \cdot \mu F-\frac{1}{4} \frac{\mu F}{K T}\right\} \\
& =\frac{K}{4}\left(\frac{\mu F}{K T}\right)^{2}
\end{aligned}
$$

\section{CLASSICALLIMITING BEHAVIOROF QUANTUM} STATISTICS

We shall now present a short account of the way that the classical limit emerges from the formulae of quantum statistics. We start by studying a free two-dimensional rotator for which

$$
Z^{o}=\sum_{m=-\infty}^{+\infty} e^{-\alpha m^{2}}
$$

where $\alpha=\beta h^{2} / 2 I$. The classical limit emerged at high temperatures $(\alpha<<1)$ when we replaced the sum by an integral and the leading term was $Z_{\text {classical }}^{0}=\sqrt{\frac{\pi}{\alpha}}$ refer to Eqn. (13).

Now we want to examine the corrections to this limit, obtaining some sort of series in $\alpha$ The sum involved in $Z^{\circ}$ is a theta function whose properties have been well studied ${ }^{[8,9]}$. We can therefore already see that there may be some difficulty in replacing the sum by an integral. For even if $\alpha$ is very small (but not zero), there will always be some value of $|m|$ beyond which the terms in the sum begin to change rapidly.

The Euler-Maclaurin formula [9] is customarily used to replace a sum by an integral and to obtain corrections. This formula states

$$
\begin{aligned}
\sum_{n=a}^{\infty} f(n)= & \int_{a}^{\infty} f(x) d x+\frac{1}{2} f(a)- \\
& \frac{1}{12} f^{1}(a)+\frac{1}{720} f^{11}(a) \ldots
\end{aligned}
$$

The coefficients are related to the well known Bernouilli numbers [9]. To apply this to our present case, we write

$Z^{o}=1+2 \sum_{m=0}^{\infty} e^{-\alpha m^{2}}=2 \sum_{m=0}^{\infty} e^{-\alpha m^{2}}-1$

Thus

$Z^{o} \Rightarrow \sqrt{\frac{\pi}{\alpha}}$

The following exact relation is known from the theory of theta functions ${ }^{[8]}$

$$
\sum_{m=-\infty}^{+\infty} e^{-\alpha m^{2}}=\sqrt{\frac{\pi}{\alpha}} \sum_{m=-\infty}^{\infty} e^{-\frac{\pi^{2} m^{2}}{\alpha}}
$$

The LHS is a rapidly converging series for $\alpha\rangle\rangle 1$, whereas the RHS expression converges rapidly for $\alpha<\langle 1$.

Thus

$Z^{o}=\sqrt{\frac{\pi}{\alpha}}\left\{1+2 e^{-\frac{\pi^{2}}{\alpha}}+2 e^{-\frac{4 \pi^{2}}{\alpha}}+\ldots ..\right\}$

where $e^{-\frac{\pi^{2}}{\alpha}}=e^{\frac{-\pi^{2} 2 I K T}{\hbar^{2}}}$ and the dependence on temperature is exponential. Thus the corrections to $Z^{o}$ do not form a power series in $h^{2}$ or $1 / K T$. The same thing is true of the thermodynamic functions such as the free energy or specific heat. The study of the corrections to the classical limit in the presence of an applied field is much more complex and is the subject of a later communication.

\section{REFERENCES}

1. Mandal, F., (1977). Statistical Physics, John Wiley and Sons, New York.

2. Terletzikii Ya P., (1971). Statistical Physics, NorthHolland Pub. Co., Amsterdam.

3. Gross, E. P. and Malo, O.J., (1972). Chem. Phys., 57, 2229. 
4. Malo, O. )1974). Nuovo Cimento, 21B, 171, (1974)

5. Gross, E. P. (1976). J. Stat. Phys., 15, 181.

6. Gross, E.P. and Lebowitz, J. (1956). Phys. Rev. 104, $152 \mathrm{~B}$.

7. Sneddon, I. N., (1956). Special Functions of Mathematical Physics and Chemistry, Oliver \& Boyd, Edinburgh.
8. Gradshteyn, I.S and Ryzhik, I. W. (1965). Table of Integrals Series and Products, Academic Press, New York (1965).

9. Boas, M. L.(1971). Mathematical Methods in the Physical Sciences, John Wiley and Sons Inc. New York (1971). 